\title{
BOUNDARY CONVERGENCE AND BOUNDARY LIMITS OF BLASCHKE PRODUCTS
}

\author{
C. N. LINDEN
}

\begin{abstract}
For a given countable subset $\gamma$ of the unit circle, a method is given for the construction of Blaschke products $B(z, A)$ which converge at all points of $\gamma$ and which, for each point $e^{i p}$ of $\gamma$, either (a) have no asymptotic value at $e^{i \phi}$ or (b) have an asymptotic value at $e^{i \phi}$ not equal to $B\left(e^{i p}, A\right)$.
\end{abstract}

1. Introduction. A sequence $A$ of complex numbers is said to be a Blaschke sequence if $A$ is a subset of the unit disc $D$ and $\sum_{\alpha \in A}(1-|\alpha|)$ converges. Associated with each Blaschke sequence $A$ is a Blaschke product

$$
B(z, A)=\prod_{\alpha \in A} b(z, \alpha)
$$

where

$$
b(z, \alpha)=\frac{|\alpha|-z e^{-i \arg \alpha}}{1-z \bar{\alpha}},
$$

and $\arg \alpha$ is defined to be $\pi$ when $\alpha=0$. The function $B(z, A)$ is regular, and bounded in modulus by 1 , in $D$. It has zeros only on the set $A$, each zero $\alpha$ having multiplicity equal to the number of times that $\alpha$ appears in $A$. The order of terms in the sequence $A$ does not affect the behaviour of $B(z, A)$ in $D$, although it may affect the convergence of $B(z, A)$ at points $z$ of $\partial D$. We abide by the usual convention that implies the monotonicity of the corresponding sequence of moduli of zeros, and we suppose that when Blaschke products are multiplied or factorised the new products that arise maintain this ordering of zeros.

It is well known $\left[5\right.$, p. 304] that the radial limit $B^{*}\left(e^{i \varphi}, A\right)=\lim _{r \rightarrow 1-} B\left(r e^{i \varphi}, A\right)$ exists and has modulus 1 for almost all $\varphi$ in $[0,2 \pi)$. Radial limits are related to the convergence of $B(z, A)$ at particular points $e^{i \varphi}$ on $\partial D$ by the fact that

$$
\sum_{\alpha \in A} \frac{1-|\alpha|}{\left|\alpha-e^{i \varphi}\right|}<\infty,
$$

which is equivalent to the absolute convergence of $B\left(e^{i \varphi}, A\right)$, is a sufficient condition for the equality $B^{*}\left(e^{i \varphi}, A\right)=B\left(e^{i \varphi}, A\right)$ to hold. Since it is known [3] that $B\left(e^{i \varphi}, A\right)$ may diverge at each point of $\partial D$ it follows that $B^{*}\left(e^{i \varphi}, A\right)$ can exist even when $B\left(e^{i \varphi}, A\right)$ diverges.

In this paper we show, in the opposite direction, that, for any sequence of points $\left\{e^{i \varphi_{m}}\right\}$ on $\partial D$, there exist Blaschke sequences $A$ and $A^{\prime}$ such that (i) $B\left(e^{i \varphi_{m}}, A\right)$ and

Received by the editors October 1, 1979.

1980 Mathematics Subject Classification. Primary 30D50; Secondary 30D35, 30D40, 30 D55. 
$B^{*}\left(e^{i \varphi_{m}}, A\right)$ both exist but are different for $m=1,2,3, \ldots$ and (ii) $B\left(e^{i \varphi_{m}}, A^{\prime}\right)$ exists while $B^{*}\left(e^{i \varphi_{m}}, A^{\prime}\right)$ does not exist for $m=1,2,3, \ldots$ The particular properties displayed by these examples may be contrasted with Abel's theorem on power series which states that if $f(z)=\sum_{n=0}^{\infty} a_{n} z^{n}$ converges for $z=e^{i \varphi}$ then the radial limit $f^{*}\left(e^{i \varphi}\right)$ exists and is equal to $f\left(e^{i \varphi}\right)$.

2. Blaschke products with asymptotic values zero on a countable set. The property (i) of $\S 1$ is a consequence of the example included in the following result.

Proposition 1. Let $\left\{\Gamma_{m}\right\}$ be a sequence of arcs in $\bar{D}$ such that $\partial D \cap \Gamma_{m}$ is a single point $e^{i \varphi_{m}}$ for $m=1,2,3, \ldots$ Then there is a Blaschke sequence $A$ such that

$$
\lim _{\substack{z \rightarrow e^{i \varphi} \\ z \in \Gamma}} B(z, A)=0
$$

while $B\left(e^{i \varphi}, A\right)$ converges (to a limit of modulus 1) for $\varphi=\varphi_{m}, \Gamma=\Gamma_{m}$ and $m=1,2,3, \ldots$.

A theorem of Lindelöf $\left[1\right.$, p. 3] implies that if $(2.1)$ holds then $B^{*}\left(e^{i \varphi}, A\right)=0$, so that (i) of $\$ 1$ follows immediately from Proposition 1. In proving Proposition 1 itself we deal with three cases separately.

(a) Let $\left\{\Gamma_{m}\right\}$ consist of just one arc $\Gamma$, meeting $\partial D$ in $e^{i \varphi}$. Then [4] there is a Blaschke sequence $A_{1}$ for which

$$
\lim _{\substack{z \rightarrow e^{i \varphi} \\ z \in \Gamma}} B\left(z, A_{1}\right)=0 .
$$

For convenience, we may obtain $A_{1}$ in the form $A_{2} \cup A_{3}$, where

$$
\begin{aligned}
& A_{2}=\left\{\alpha \in A_{1}: \sin (\varphi-\arg \alpha)>0\right\}, \\
& A_{3}=\left\{\alpha \in A_{1}: \sin (\varphi-\arg \alpha)<0\right\},
\end{aligned}
$$

at least one of the sets $A_{2}$ and $A_{3}$ being nonempty (see also [6]). It may be assumed, without loss of generality, that the moduli of the members of $A_{1}$ are distinct, and that

$$
\lim _{\substack{|\alpha| \rightarrow 1 \\ \alpha \in A_{j}}} \frac{1-|\alpha|}{\mid \alpha-e^{i \varphi \mid}}=0
$$

for $j=2$ and 3 .

The Blaschke sequence

$$
\mathcal{Q}(\varphi)=A_{1} \cup\left\{\bar{\alpha} e^{2 i \varphi}: \alpha \in A_{1}\right\}
$$

is defined with its elements arranged so that, as usual, their moduli form a monotonic increasing sequence. Let $\mathbb{Q}(\varphi, m)$ and $A(m)$ denote the first $m$ terms of $Q(\varphi)$ and $A_{1}$ respectively. Then

$$
B\left(e^{i \varphi}, Q(\varphi, 2 m)\right)=\prod_{\alpha \in A(m)} \frac{\left(|\alpha|-e^{i(\varphi-\arg \alpha)}\right)\left(|\alpha|-e^{-i(\varphi-\arg \alpha)}\right)}{\left(1-\bar{\alpha} e^{i \varphi}\right)\left(1-\alpha e^{-i \varphi}\right)}=1 .
$$


But if $\alpha \in A_{1}$, we have [3] that

$$
\frac{|\alpha|-e^{i(\varphi-\arg \alpha)}}{1-\bar{\alpha} e^{i \varphi}}=e^{-2 i \psi},
$$

where

$$
\psi=\arctan \left(\frac{1-|\alpha|}{1+|\alpha|}\right) \cot \frac{1}{2}(\varphi-\arg \alpha) \rightarrow 0
$$

as $|\alpha| \rightarrow 1$ by (2.3). It follows, on taking limits, that $B\left(e^{i \varphi}, \mathbb{Q}(\varphi)\right)=1$. Since the equality

$$
\lim _{\substack{z \rightarrow e^{i \varphi} \\ z \in \Gamma}} B(z, \mathcal{Q}(\varphi))=0
$$

follows immediately from (2.2) and (2.4), we have Proposition 1 in the case where $\left\{e^{i \varphi_{m}}\right\}$ is a single point $e^{i \varphi}$ and $A=\mathscr{Q}(\varphi)$.

(b) When the sequence $\left\{e^{i \varphi_{m}}\right\}$ is finite, sequences $Q\left(\varphi_{m}\right)$ can be constructed as in case (a). The sequence $A=\cap_{m} \mathbb{Q}\left(\varphi_{m}\right)$ is easily seen to satisfy the requirements of Proposition 1 , since $B\left(e^{i \varphi}, \mathcal{Q}(\varphi)\right)$ converges everywhere on $\partial D$.

(c) It remains to consider the case where $\left\{e^{i \varphi_{m}}\right\}$ is an infinite sequence. For each $m$, the sequence $Q\left(\varphi_{m}\right)$ can be constructed according to case (a). Let $A(m, p)$ denote the Blaschke sequence formed by deleting the first $p$ terms of $Q\left(\varphi_{m}\right)$. Then, for each $p,(2.1)$ holds for $A=A(m, p), \varphi=\varphi_{m}$ and $\Gamma=\Gamma_{m}$, while $B\left(e^{i \varphi_{m}}, A(m, p)\right)$ converges. We choose $p=p(m)$ by induction so that for given $m$

$$
\sum_{\alpha \in A(m, p)}(1-|\alpha|)<\frac{M(m)}{\pi 2^{m}},
$$

where $0<M(m)=\inf _{1<j<m}\left|\varphi_{m}-\varphi_{j}\right|$, and the closed convex hulls of $A(m, p(m))$ and $A(j, p(j))$ are disjoint for $j=1,2, \ldots, m-1$. We also impose the condition that

$$
\left|\varphi_{j}-\arg \alpha\right|>\frac{1}{2}\left|\varphi_{j}-\varphi_{m}\right| \quad(\alpha \in A(m, p(m)))
$$

for $j=1,2, \ldots, m-1$.

The inequality (2.5) implies that $A=\cup_{m=1}^{\infty} A(m, p(m))$ is a Blaschke sequence. Since the construction of $A$ shows that (2.1) holds for $\varphi=\varphi_{m}, \Gamma=\Gamma_{m}, m=$ $1,2,3, \ldots$, we have only to prove that $B\left(e^{i \varphi_{m}}, A\right)$ converges for $m=1,2,3, \ldots$ To this end we consider the factorisation

$$
B(z, A)=B_{1, m}(z, A) B_{2, m}(z, A)
$$

where

$$
\begin{aligned}
& B_{1, m}(z, A)=\prod_{k=1}^{m} B(z, A(k, p(k))), \\
& B_{2, m}(z, a)=\prod_{k=m+1}^{\infty} B(z, A(k, p(k))),
\end{aligned}
$$

the zeros of each of the two products being ordered in the usual way.

Case (b) shows that $B_{1, m}\left(e^{i \varphi_{m}}, A\right)$ converges (though not absolutely). It will be shown that, on the other hand, the product $B_{2, m}\left(e^{i \varphi_{m}}, A\right)$ does converge absolutely. 
We note first that

$$
b\left(e^{i \varphi}, \alpha\right)=1-\frac{\left(1+e^{i(\varphi-\arg \alpha)}\right)(1-|\alpha|)}{1-\bar{\alpha} e^{i \varphi}} \quad(0<|\alpha|<1,0<\varphi<2 \pi),
$$

where

$$
\left|1-\bar{\alpha} e^{i \varphi}\right|^{2}=(1-|\alpha|)^{2}+4|\alpha| \sin ^{2} \frac{1}{2}(\varphi-\arg \alpha) .
$$

The choice of the sequence $\{p(m)\}$ implies that

$$
\begin{aligned}
\frac{1-|\alpha|}{\left|1-\bar{\alpha} e^{i \varphi_{m}}\right|} & <\frac{(1-|\alpha|)|\alpha|^{-1 / 2}}{2\left|\sin \frac{1}{2}\left(\varphi_{m}-\arg \alpha\right)\right|} \\
& <\frac{\pi(1-|\alpha|)|\alpha|^{-1 / 2}}{\left|\varphi_{m}-\varphi_{k}\right|} \quad(\alpha \in A(k, p(k)))
\end{aligned}
$$

for $k>m$. Since (2.5) implies that $|\alpha|>\frac{1}{2}$ we have

$$
\sum_{\alpha \in A(k, p(k))} \frac{1-|\alpha|}{\mid 1-\bar{\alpha} e^{i \varphi_{m} \mid}}<2^{1 / 2-k}
$$

The absolute convergence of $B_{2, m}\left(e^{i \varphi_{m}}, A\right)$ follows immediately, and the proof of Proposition 1 is complete.

3. Blaschke products without radial limits on a countable set. The method used in $\$ 2$ can be extended to establish the assertion (ii) of $\$ 1$. We note the amendments necessary to prove the following result.

Proposition 2. Given a sequence $\left\{e^{i \varphi_{m}}\right\}$ on $\partial D$ there is a Blaschke sequence $A^{\prime}$ such that $B\left(e^{i \varphi_{m}}, A^{\prime}\right)$ converges but $B^{*}\left(e^{i \varphi_{m}}, A^{\prime}\right)$ does not exist for $m=1,2,3, \ldots$

Essentially we need to establish Proposition 2 in the case where $\left\{e^{i \eta_{m}}\right\}$ is a single point $e^{i \varphi}$. Let $S$ denote the sequence of points

$$
\left\{a_{n, k}: 1<k<n, n=1,2,3, \ldots\right\},
$$

where

$$
a_{n, k}=\left(1-\frac{p(n)}{n+k}\right) e^{i(\varphi+p(n))} \quad(1<k<n, n=1,2,3, \ldots)
$$

and $p(n)=2^{-n^{2}}$. It will be shown that

$$
\liminf _{r \rightarrow 1-}|B(r, S)|<e^{-1 / 8}<\limsup _{r \rightarrow 1-}|B(r, S)|=1 .
$$

Let $n$ be a given positive integer, and $r_{N}=1-2^{-N(N+1)}$. If $n<N$ then

$$
\begin{aligned}
\left|b\left(r_{N} e^{i \varphi}, a_{n, k}\right)\right| & >\frac{r_{N}-\left|a_{n, k}\right|}{1-r_{N}\left|a_{n, k}\right|}>\frac{r_{N}-\left|a_{n, k}\right|}{2-r_{N}-\left|a_{n, k}\right|} \\
& =\frac{1-(n+k) 2^{-N(N+1)} / p(n)}{1+(n+k) 2^{-N(N+1)} / p(n)} \\
& >\left(1-\frac{(n+k) 2^{-N(N+1)}}{p(n)}\right)^{2}
\end{aligned}
$$


for $1<k<n$. Therefore

$$
\begin{aligned}
\prod_{n=1}^{N} \prod_{k=1}^{n}\left|b\left(r_{N} e^{i \varphi}, a_{n, k}\right)\right| & >\prod_{n=1}^{N}\left(1-n p(N) 2^{1-N} / p(n)\right)^{2 n} \\
& >\left(1-N 2^{1-N}\right)^{2 N^{2}} \rightarrow 1
\end{aligned}
$$

as $N \rightarrow \infty$. On the other hand, if $n \geqslant N+1$, we can prove similarly that

$$
\left|b\left(r_{N} e^{i \varphi}, a_{n, k}\right)\right|>\left(1-p(n) 2^{N(N+1)} /(n+k)\right)^{2}>\left(1-2^{-n} n\right)^{2} .
$$

Thus

$$
\begin{aligned}
\prod_{n=N+1}^{\infty} \prod_{k=1}^{n}\left|b\left(r_{N} e^{i \varphi}, a_{n, k}\right)\right| & >\prod_{n=N+1}^{\infty}\left(1-2^{-n} / n\right)^{2 n} \\
& >1-\sum_{n=N+1}^{\infty} 2^{1-n}=1-2^{1-N} \rightarrow 1
\end{aligned}
$$

as $N \rightarrow \infty$. This proves the equality asserted in (3.1).

In order to complete the proof of (3.1) we consider $B\left(t_{N} e^{i \varphi}, S\right)$, where $t_{N}=1-$ $2^{-N^{2}}$. Then for $1 \leqslant k \leqslant N$ we have

$$
\left|b\left(t_{N} e^{i \varphi}, a_{N, k}\right)\right|^{2}=\frac{\left(t_{N}-\left|a_{N, k}\right|\right)^{2}+4\left|a_{N, k}\right| t_{N} \sin ^{2}\left(2^{-1-N^{2}}\right)}{\left(1-t_{N}\left|a_{N, k}\right|\right)^{2}+4\left|a_{N, k}\right| t_{N} \sin ^{2}\left(2^{-1-N^{2}}\right)}
$$

where

$$
1-t_{N}\left|a_{N, k}\right|=p(N)\left(1+\frac{1-p(N)}{N+k}\right)>p(N) .
$$

Hence

$$
\begin{aligned}
\left|b\left(t_{N} e^{i \varphi}, a_{N, k}\right)\right|^{2} & <\frac{(1-1 /(N+k))^{2}+1}{2} \\
& <1-\frac{1}{2(N+k)}<1-\frac{1}{4 N}
\end{aligned}
$$

from which we obtain

$$
\left|B\left(t_{N} e^{i \varphi}, S\right)\right|<\prod_{k=1}^{N}\left|b\left(t_{N} e^{i \varphi}, a_{N, k}\right)\right|<\left(1-\frac{1}{4 N}\right)^{N / 2}<e^{-1 / 8}
$$

for $N=1,2,3, \ldots$. This completes the proof of (3.1).

The sequence $A^{\prime}$ is now defined as $S \cup\left\{\bar{\alpha} e^{2 i \varphi}: \alpha \in S\right\}$. The relation (3.1) implies that

$$
\liminf _{r \rightarrow 1_{-}^{-}}\left|B\left(r e^{i \varphi}, A^{\prime}\right)\right|<e^{-1 / 4}<\limsup _{r \rightarrow 1-}\left|B\left(r e^{i \varphi}, A^{\prime}\right)\right|=1,
$$

so that $B^{*}\left(e^{i \varphi}, A^{\prime}\right)$ does not exist. Since the elements of $A^{\prime}$ are situated symmetrically about the radius of $D$ through $e^{i \varphi}$, and

$$
\lim _{\substack{|\alpha| \rightarrow 1 \\ \alpha \in A^{\prime}}} \frac{1-|\alpha|}{\left|\alpha-e^{i \varphi}\right|}=0
$$


the proof of Proposition 1 (a) can be readily adapted to show that $B\left(e^{i \varphi}, A^{\prime}\right)$ converges. Thus we have proved Proposition 2 in the case when $\left\{e^{i \varphi_{m}}\right\}$ consists of just one point. The proofs in other cases can be effected by applying the methods of proof (b) and (c) for Proposition 1.

Finally, we note that the absolute convergence of the product $B_{2, m}\left(e^{i \varphi}, A\right)$ in $\S 2$, and a corresponding property associated with the Blaschke product of Proposition 2 , allow the two types of boundary limit property considered in $\$ \S 2$ and 3 to be combined. Thus, for a given pair of nonintersecting sequences $\left\{e^{i \varphi_{m}}\right\}$ and $\left\{e^{i \theta_{m}}\right\}$ on $\partial D$, and a sequence of arcs $\left\{\Gamma_{m}\right\}$ in $\bar{D}$ such that $\partial D \cap \Gamma_{m}=e^{i \varphi_{m}}$ for $m=$ $1,2,3, \ldots$, there is a Blaschke sequence $A^{\prime \prime}$ such that $B\left(z, A^{\prime \prime}\right)$ converges at each point $\left\{e^{i \varphi_{m}}\right\}$ and $\left\{e^{i \theta_{m}}\right\}$, while

$$
\lim _{\substack{z \rightarrow e^{i \varphi} \\ z \in \Gamma}} B\left(z, A^{\prime \prime}\right)=0
$$

for $e^{i \varphi}=e^{i \varphi_{m}}, \Gamma=\Gamma_{m}$ and $m=1,2,3, \ldots$, and $B^{*}\left(z, A^{\prime \prime}\right)$ does not exist at any point of $\left\{e^{i \theta_{m}}\right\}$.

\section{REFERENCES}

1. M. L. Cartwright, Integral functions, Universtity Press, Cambridge, 1956.

2. O. Frostman, Sur les produits de Blaschke, Kungl. Fysiogr. Sällsk. i Lund Förh. 12 (1942), No. 15, 169-182.

3. C. N. Linden, On Blaschke products diverging everywhere on the boundary of the unit disc, Proc. Amer. Math. Soc. 55 (1976), 62-64.

4. C. N. Linden and H. Somadasa, Zero tracts of Blaschke products, Canad. J. Math. 18 (1966), 1072-1078.

5. W. Rudin, Real and complex analysis, McGraw-Hill, New York, 1974.

6. M. L. Weiss, Note on a theorem of Beurling, Proc. Amer. Math. Soc. 19 (1968), 793-795.

Department of Pure Mathematics, University College of Swansea, Swansea, Wales, Great BRITAIN 